\title{
Sperm cryopreservation and in vitro fertilization techniques for the African turquoise killifish Nothobranchius furzeri
}

\section{Luca Dolfi}

Max Planck Institute for Biology of Ageing

\section{Tsz Kin Suen}

Max Planck Institute for Biology of Ageing

Roberto Ripa

Max Planck Institute for Biology of Ageing

Adam Antebi ( $\square$ aantebi@age.mpg.de )

Max Planck Institute for Biology of Ageing

\section{Research Article}

Keywords: African turquoise killifish, Nothobranchius furzeri, embryology, ageing

Posted Date: March 17th, 2021

DOl: https://doi.org/10.21203/rs.3.rs-296488/v1

License: (c) (i) This work is licensed under a Creative Commons Attribution 4.0 International License. Read Full License 
$5 \quad$ Luca Dolfi ${ }^{1}$, Tsz Kin Suen ${ }^{1}$, Roberto Ripa ${ }^{1}$, Adam Antebi $^{1,2^{*}}$

$8{ }^{1}$ Max Planck Institute for Biology of Ageing, Cologne, Germany

$9 \quad{ }^{2}$ Cologne Excellence Cluster on Cellular Stress Responses in Aging Associated

10 Diseases (CECAD), University of Cologne, Cologne, Germany

$11{ }^{*}$ corresponding author

$12 \quad$ E-mail: aantebi@age.mpg.de 


\section{Abstract}

14 Over the last decade, the African turquoise killifish, Nothobranchius furzeri, has emerged as an important model system for the study of vertebrate biology and ageing. $N$. furzeri propagation, however, can pose challenges due to the short window of fertility, the efforts and space requirements involved in continuous strain maintenance, and the risks of inbreeding. The current method for long

19 term strain preservation relies on arrest of embryos in diapause. Though useful,

20 it remains unclear how long embryos can survive in this state. To create an

21 alternative for long term maintenance, we developed a robust protocol to

22 cryopreserve and revive sperm for in vitro fertilization (IVF). We tested a variety

23 of extender and activator buffers for sperm IVF, as well as cryoprotectants to 24 achieve feasible long-term storage and fertilization conditions tailored to this 25 species. Our protocol enabled sperm to be preserved in a cryogenic condition for 26 months and to be revived an average of $40 \%$ viability upon thawing. Thawed

27 sperm were able to fertilize nearly the same number of eggs as natural

28 fertilization, with an average of $\sim 25 \%$ and peaks of $\sim 55 \%$ fertilization. This

29 technical advance will greatly facilitate the use of $N$. furzeri as a model organism.

31 Abbreviations used:

32 HBSS: Hank's Balanced Salt Solution

33 BSMIS: Buffered sperm motility-inhibiting solution

34 FBS: Fetal bovine serum 
DMSO: Dimethyl sulfoxide

36 DMF: Dimethylformamide

37 MetOH: Methanol

38 DMA: Dimethylacetamide

39 IVF: In vitro fertilization

41 Introduction

42 Over the last few years, the African killifish Nothobranchius furzeri has

43 emerged as an important model system for the study of vertebrate ageing. $N$.

44 furzeri's life cycle is characterized by a fast growth rate, reaching sexual maturity

45 by $4-5$ weeks, and a maximum lifespan of $6.5-7$ months in the wild type strain

46 GRZ-AD ${ }^{1,2}$, making this fish among the shortest-lived vertebrate species bred in

47 captivity and a unique platform for the rapid exploration of ageing and age-

48 associated diseases ${ }^{3}$.

Rapid growth, maturation and ageing carry drawbacks, however, which

50 make maintenance in captivity of $N$. furzeri challenging. It leads to a swift

51 passing of generations, which makes it difficult to maintain a primary founder

52 genotype and prevent inbreeding ${ }^{4,5}$. Fertility is limited to the $5^{\text {th }}-20^{\text {th }}$ week and

53 maximal between the $6^{\text {th }}-11^{\text {th }}$ weeks ${ }^{6-8}$ of life, giving a narrow breeding window,

54 whereas in other fish species the breeding window can correspond to years ${ }^{9}$.

55 Importantly, the success of transposon-mediated transgenesis ${ }^{10}$ and CRISPR-

56 mediated mutagenesis ${ }^{11}$ in Nothobranchius means that more genetically 
engineered lines require continuous maintenance and space usage. Breeding to preserve a line takes significant effort and carries the risk of accident or infection that can result in strain loss. Furthermore, $N$. furzeri husbandry requires considerable space, since they are often optimally grown when singly housed because of fish-to-fish harassment and food competition ${ }^{8}$.

Given these constraints, it is essential to develop protocols to maintain stocks without constant breeding. Currently, the main way to maintain strains is through diapause, a state of arrested development ${ }^{2}$. To date, it is difficult to induce and release diapause in a controlled and synchronized manner from a large pool of embryos ${ }^{12,13}$, though recently vitamin $D$ has been shown to regulate diapause in other killifish species ${ }^{12}$. Moreover, eggs in diapause need periodic maintenance, since the medium and substrate must be checked, cleaned and changed ${ }^{8}$.As the number of strains and lines increase, this method becomes untenable at larger scales.

To solve these problems, fish researchers rely on sperm cryopreservation and in vitro fertilization techniques. Through cryopreservation, fish genetic pools can be stored in sperm banks with minimum maintenance effort for years. Upon revival, thawed sperm can usually fertilize eggs with a success rate from $10-80 \%$ ${ }^{14}$. Specific protocols have been established to preserve and activate the sperm of various production and research species ${ }^{15}$. To date, however, there is no established protocol available for sperm cryopreservation and in vitro fertilization for killifish species. Here we developed a feasible protocol for Killifish sperm cryopreservation and in vitro fertilization. Our method will facilitate the husbandry and the usefulness of $N$. furzeri as a model organism for research. 


\section{Results}

\section{Extender and Activator}

The optimum conditions for spermatozoa cryopreservation and in vitro fertilization can vary greatly for each fish species. For most freshwater fish,

sperm motility is initiated by hypotonic osmolalities ${ }^{16}$ and/or by alteration of ion concentration such as potassium or calcium ${ }^{17,18}$. Once activated, the sperm exhibit a short period of motility (30 s to $5 \mathrm{~min}$ ), depending on species ${ }^{16}$.

Protocols from other fish species make use of an "extender" and an "activator" solution. The extender is usually a high molality saline-buffered solution mixed with the extracted sperm that keeps them stable and inactive, and is comparable to the ion concentrations inside the gonad ${ }^{14,19}$. By contrast, the activator is a low molality solution, which when added to the sperm-extender solution, initiates sperm activity and movement. In some cases, the activator is simply a dilution of the extender. In addition, specific concentrations of ions like potassium, calcium and magnesium are required for proper sperm activation, motility, directionality and endurance ${ }^{14,20}$.

For Zebrafish and Medaka there are several useful existing protocols. These typically use various solutions, such as Hank's Balanced Salt Solution (HBSS), Buffered sperm motility-inhibiting solution (BSMIS) or Fetal bovine serum (FBS) as extender. A dilution of these solutions or the addition of another composite salt, such as Instant Ocean or Iwamatsu solution, trigger activation $15,21,22$. Our first goal therefore was to test sperm activation using these known solutions. Sperm was collected from male fish between 9 and 11 weeks of age through dissection and gonad isolation. Before dissection, fish were carefully 
105 dried to prevent spontaneous sperm activation due to the presence of water.

106 Testes were placed into various solutions (extenders at various dilutions), in an

107 Eppendorf tube and quickly shaken for 5-10 seconds to release sperm into the 108 medium. We tested tank water, deionized water, BSMIS, FBS, HBSS and 109 Iwamatsu solution at different dilutions and molalities (Figure 1). 

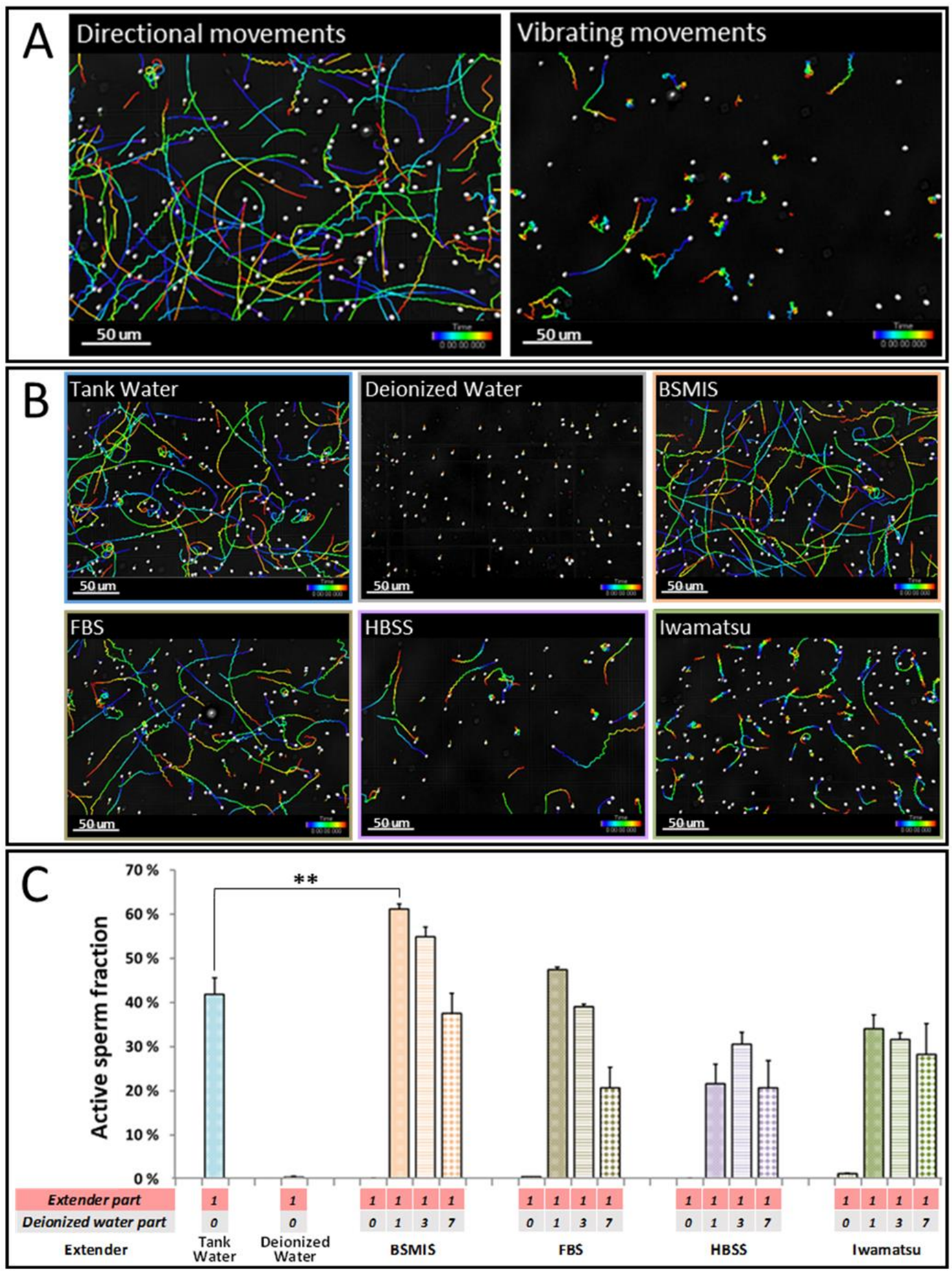

112 Figure 1: Fresh sperm activation in different buffers. (A) Motility tracing of different types of sperm movement that

113 occur after activation, resulting in proper directional movements (left) or erratic inefficient vibrating movements

114 (right). (B) Representative images of sperm movement in activated by 1:1 dilution of the indicated buffer conditions

115 (C) Quantification of sperm activation in different buffers calculated as the average of number of active sperm

116 divided by total sperm among biological replicates. Asterisks indicate solutions that are significantly more effective 
120 Immediately afterwards, the gonads were removed from the medium, $10 \mu \mathrm{l}$ of the

121 mixture was put in a hemocytometer chamber and sperm movements were

122 video-recorded under a microscope. The videos were subsequently analyzed

123 with Imaris ${ }^{\circledR}$ and the percentage of directionally moving spermatozoa measured

124 (Figure 1A,B,C).

Tank water, the natural medium where fish biologically breed, was able to 126 activate $\sim 40 \%$ of the sperm. Deionized water was able to trigger an initial 127 activation, which faded within seconds (data not shown), resulting immediately 128 afterwards in vibrating or immobile sperm. Stock solutions of BSMIS, FBS, HBSS 129 and Iwamatsu solutions kept the sperm inactive (Figure 1C, Extender part 1, 130 deionized water part 0), but upon dilution (Figure 1C, extender part 1, deionized 131 water parts $>0$ ) could activate sperm. Some BSMIS and FBS dilutions had 132 activation rates comparable to tank water, while BSMIS diluted with 1 part of 133 deionized water had significantly higher activation compared to tank water. 134 HBSS and Iwamatsu solutions were not significantly better than tank water as 135 activators at any dilution.

FBS is often used as an extender, which when diluted with other solutions 137 activates sperm ${ }^{21,23,24}$. Aoki et al. used FBS, and then added 2 volumes of 138 Iwamatsu solution to promote directional activation in Medaka, the species 139 closest to Nothobranchius among model fish. We therefore tested FBS buffer as 

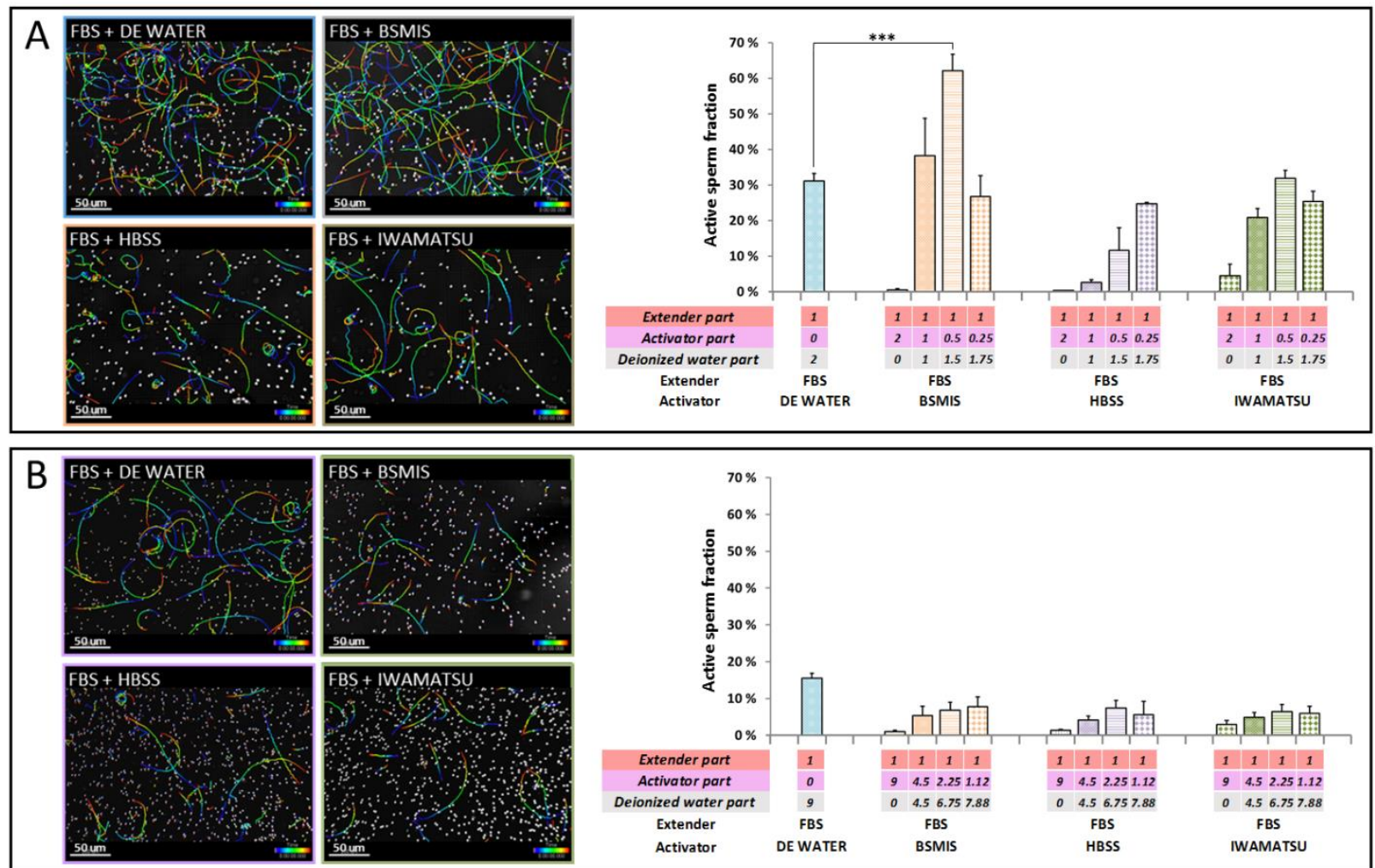

Figure 2: Fresh sperm activation using FBS as extender and different buffers for dilution. (A) Image tracking of sperm movements and quantification of directional sperm in different activating solutions, mixed in a ratio 1 to 3 with the FBS extender solution (1 volume of extender solution plus 2 volumes of activating solution at different dilutions). Ratios of extender, activator, and water for each mix are shown below each column. (B) Image tracking of sperm movements and quantification of directional sperm in different activating solutions, mixed in a ratio of 1 to 10 with the FBS extender solution (1 volume of extender solution plus 9 volumes of activating solution at different dilutions). Ratios of extender, activator, and water for each mix are shown below each column. Asterisks indicate solutions significantly more effective than tank water in activating sperm, based on a two way Anova post hoc Dunnett's test. ${ }^{*} \mathrm{p}<0.05,{ }^{* *} \mathrm{p}<0.01,{ }^{* * *} \mathrm{p}<0.001 . \mathrm{N}=3$ biological replicates for each condition.

152 Among the combinations, we observed the highest activation using FBS extender together with a dilution 1:4 of BSMIS (i.e. 1 part of FBS mixed with 0.5 parts of BSMIS and 1.5 parts of deionized water), reaching yields of $>60 \%$. In 
155 contrast to Medaka, FBS mixed with Iwamatsu did not give appreciable 156 activation of Nothobranchius sperm. In another set of experiments, we 157 increased the dilution ratio between the extender (FBS) and the activators, from 1581 plus 2 volumes respectively to 1 plus 9 volumes, respectively (Figure 2B). 159 However, the activation rates obtained in these experiments (Figure 2B) were 160 significantly worse than the previous test (Figure $2 \mathrm{~A}$ ), and most of the 161 spermatozoa were vibrating or not moving at all.

\section{Cryoprotection, Freezing and Thawing}

For sperm to survive freezing and kept long-term in a cryostatic condition, 164 the extender has to be supplemented with a cryoprotectant. These chemicals 165 surround sperm cells and prevent the formation of ice crystals that compromise 166 membrane integrity, thereby protecting them from cryodamage ${ }^{25}$. The most 167 commonly cryoprotectants used are DMSO, DMF, MetOH, glycerol, and DMA, 168 with concentrations usually set around $10 \%$ of the volume ${ }^{15,26}$. Since FBS and 169 BSMIS worked best as extenders (Figure 1C, 2A), we performed our cryostatic experiments using these solutions. We mixed the selected extender with $10 \%$ of 171 DMSO, DMF, MetOH, DMA or glycerol. Sperm was released in pre-mixed 172 extender-cryoprotectant solutions (Figure 3C) and the mixture was incubated for $1731 \mathrm{~h}$ at $4^{\circ} \mathrm{C}$ (Figure 3D), allowing the cryoprotectants to be evenly absorbed by the 174 sperm cells. Samples were then aliquoted in 60ul volumes (Figure 3E), frozen in 175 dry ice (Figure 3F), and nitrogen gas phase in sequence (Figure 3G), and stored 176 in liquid nitrogen (Figure $3 \mathrm{H}$ ). 


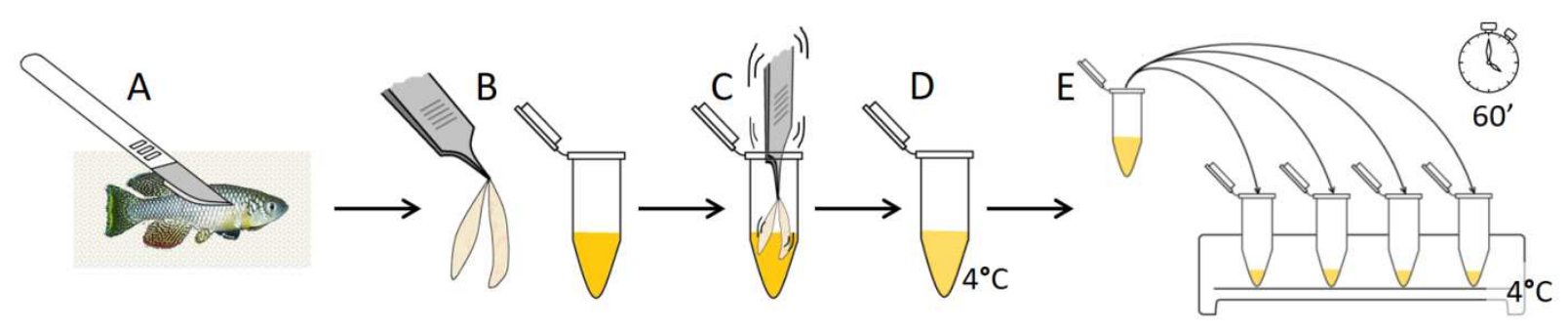

F

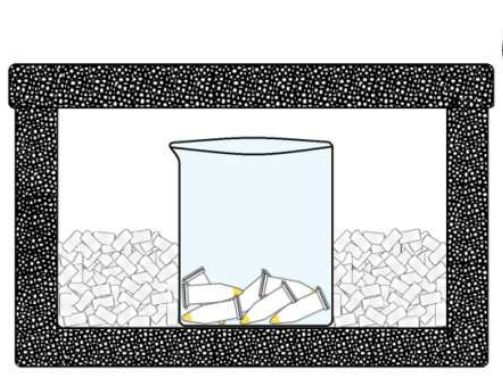

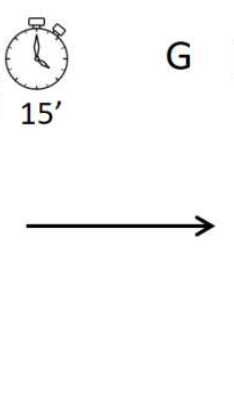
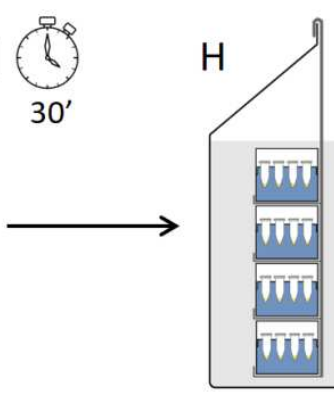

Figure 3: Schematic of sperm collection and freezing procedure. (A) Male dissection. (B) Gonad extraction. (C)

Gonads are spun in extender plus cryoprotectant solution. (D) Cryoprotectant incubation with sperm cells at $4^{\circ} \mathrm{C}$. (E)

Solution transfer in smaller aliquots to vials. (F) First freezing step setup with vials laying on the bottom of a glass

beaker surrounded by dry ice in a closed styrofoam box. (G) Second freezing step setup with vials exposed to nitrogen vapor. (H) Long term storage of vials in liquid nitrogen. For a detailed explanation, see text.

183 After $24-48 \mathrm{~h}$ freezing, the sperm were revived through rapid thawing in a $30^{\circ} \mathrm{C}$ water bath (see optimization below) and activated by the addition of 2 volumes of 185 BSMIS (diluted 1:4 in the case of FBS extender and 1:2 in the case of BSMIS extender). Overall we found that sperm cryopreserved using $10 \%$ DMSO as cryoprotectant maintained the highest level of activation (Figure 4A), followed by 188 methanol (in FBS extender only). DMF, Glycerol and DMA were ineffective at cryoprotection, resulting in immobile or inviable sperm upon thawing. 

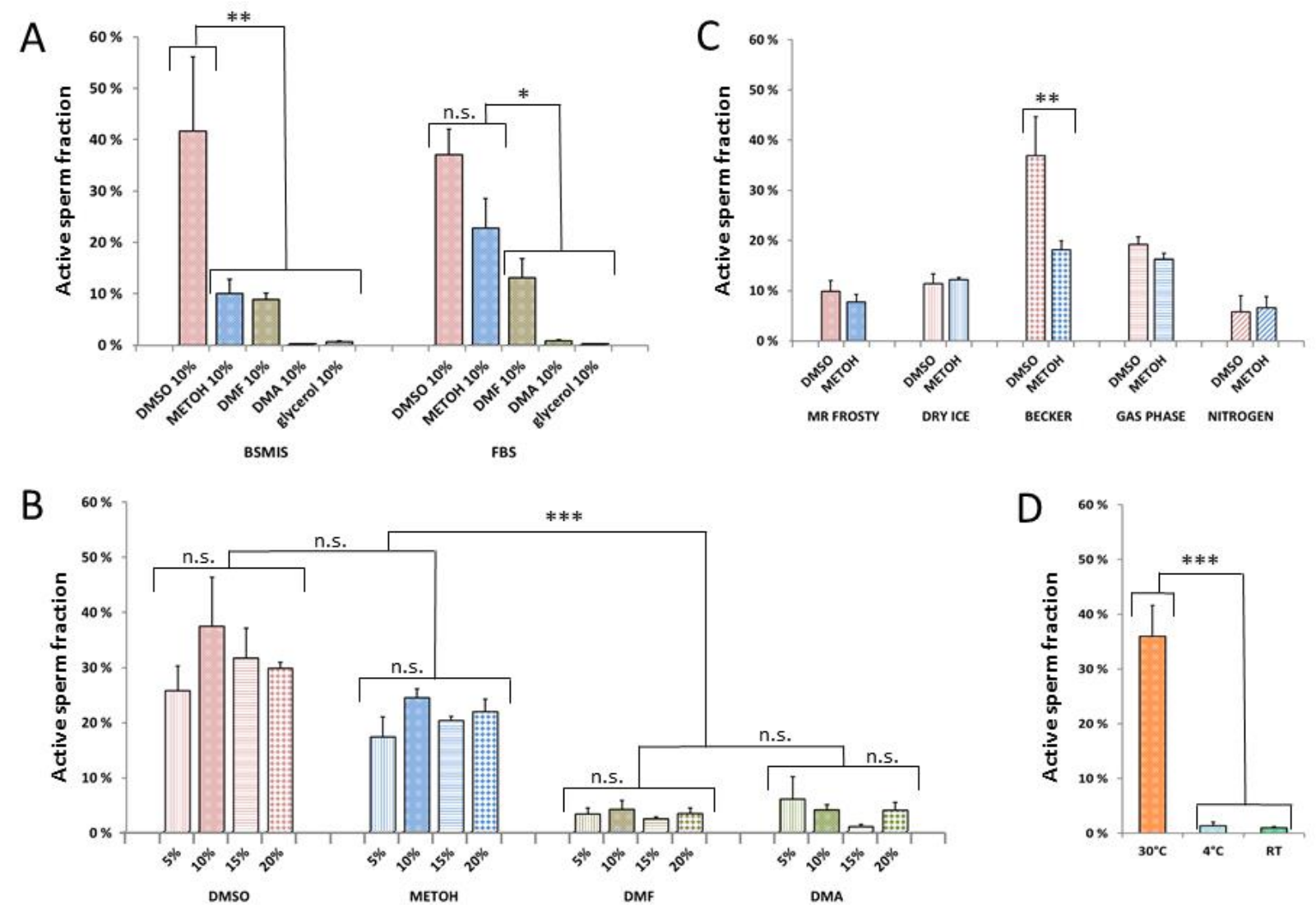

Figure 4: Cryoprotectants, cryopreservation and thawing efficiencies. (A) Effect of different cryoprotectants on

sperm activation using BSMIS and FBS extender solutions. Y bar represents the portion of sperm able to reactivate upon thawing. Statistical analysis used is two way Anova with multiple comparison Tukey's post hoc correction. In BSMIS extender, DMSO performed significantly better compared to the other cryoprotectants. In FBS extender, DMSO and MetOH performed significantly better compared to the other cryoprotectants .(B) Effect of different concentrations of cryoprotectants in FBS extender. Asterisks indicate significance levels of two way Anova multiple comparison test with post hoc Dunnett's correction. ${ }^{*} p<0.05,{ }^{* *} p<0.01, * * * p<0.001$ (C) Different freezing methods with different cooling rates applied to FBS extender plus $10 \%$ cryoprotectant. Asterisks indicate significance levels of two way Anova multiple comparison test with post hoc Sidak's correction. ${ }^{*} p<0.05, * * p<0.01, * * * p<$ 0.001 (D) Different thawing methods $\left(30^{\circ} \mathrm{C}\right.$ water bath, $4^{\circ} \mathrm{C}$ refrigerator, $\mathrm{RT}$, room temperature) with different thawing rates applied to FBS extender plus 10\% DMSO. Asterisks indicate significance levels of one way Anova multiple comparison test with post hoc Tukey's correction. ${ }^{*} p<0.05,{ }^{*} p<0.01,{ }^{* * *} p<0.001 . N=3$ biological replicates for each condition. 
204 Several concentrations of cryoprotectants were further tested in combination with 205 FBS. Sperm cryopreserved in FBS with 10 to $20 \%$ DMSO maintained 206 comparably high activation ranging from 30-37\% (Figure 4B), dropping slightly at 207 higher concentrations. Methanol was able to moderately protect sperm from 208 cryodamage at several concentrations, giving sperm activation of ca. $20 \%$. DMA 209 or DMF gave less than 10\% sperm revival at any concentration (Figure 4B).

210 Since $>20 \%$ sperm survival and activation were observed in the samples with 211 DMSO and methanol at $10 \%$, we used these concentrations of cryoprotectants 212 for further experiments.

213 The freezing procedure is also a crucial step for cryopreservation. Slow freezing 214 rates can produce large ice crystals that damage cellular ultrastructure, whereas 215 rapid freezing rates induce smaller intracellular ice crystals that are less likely to 216 trigger damage ${ }^{27}$. We therefore used various setups to establish different rates 217 of freezing. We placed the Eppendorf tubes in 1) Mr. Frosty ${ }^{\mathrm{TM}}$ Freezing 218 Container (estimated $-10^{\circ} \mathrm{C}$ per $\left.\min \right), 2$ ) a glass beaker surrounded by dry ice 219 (estimated $-20^{\circ} \mathrm{C}$ per min), 3) direct contact with dry ice (estimated $-50^{\circ} \mathrm{C}$ per $220 \mathrm{~min}$ ), 5) a Dewar vessel partially filled with liquid nitrogen, where the Eppendorf 221 tubes were placed in a box without direct contact with liquid nitrogen but exposed 222 to nitrogen gas phase (estimated $-100^{\circ} \mathrm{C}$ per min); and 6) direct liquid nitrogen 223 contact (estimated $-200^{\circ} \mathrm{C}$ per min) (Figure $4 \mathrm{C}$ ). Cooling rates were estimated 224 using a digital thermometer with a detection range $-50^{\circ} \mathrm{C} /+110^{\circ} \mathrm{C}$ with the 225 probe inserted in the tube, measuring the temperature drop divided by the time 226 required to reach $-50^{\circ} \mathrm{C} .15$ minutes after a temperature below $-50^{\circ} \mathrm{C}$ was 227 reached, sperm vials from the various freezing setups were stored in liquid 228 nitrogen overnight. After one day, frozen sperm samples were revived using 
229 BSMIS 1:4 and monitored for activation. Sperm vials frozen in a beaker

230 surrounded by dry ice $\left(-20^{\circ} \mathrm{C}\right.$ per $\left.\mathrm{min}\right)$ achieved the highest activation upon

231 revival. Sperm cryoprotected with 10\% DMSO had higher activation than those

232 cryoprotected with 10\% methanol (Figure 4C), similar to above. Therefore, we

233 selected DMSO as the final cryoprotectant and a beaker surrounded by dry ice

234 for our cryopreservation protocol.

To assess the effect of different thawing rates on the frozen sperm, we warmed the vials at 1) $30^{\circ} \mathrm{C}$ in a water bath, 2) room temperature or 3) $4^{\circ} \mathrm{C}$ in the 237 refrigerator, to achieve rapid, medium, or slow thawing rates, respectively. We 238 then revived sperm using BSMIS 1:4 and observed the sperm activation rate. We 239 found that rapid thawing in a $30^{\circ} \mathrm{C}$ water bath achieved the highest survival and 240 activation of sperm (Figure 4D).

\section{Egg fertilization and survival}

We next tested if the active sperm obtained with our protocol was able to 244 fertilize eggs obtained from $N$. furzeri females. To perform this experiment in the 245 most comparable way, we used the same males for natural and in vitro 246 fertilization. We set up several aquarium tanks with 5 females and 2 males per 247 tank and allowed them to breed naturally for 2 days. The age of the animals used 248 for fertilization were typically $9-11$ weeks of age. We then collected the eggs 249 generated from the natural breeding and monitored their survival rate until mid 250 somitogenesis. After the natural breeding, the same males were separated from 251 the females, kept alone for 2 days, and then sacrificed for gonad extraction 
252 (Figure 3A-B). The females were anesthetized, dried carefully (Figure 5A), and 253 their unfertilized eggs gently pushed out from their ovaries (Figure 5B). 


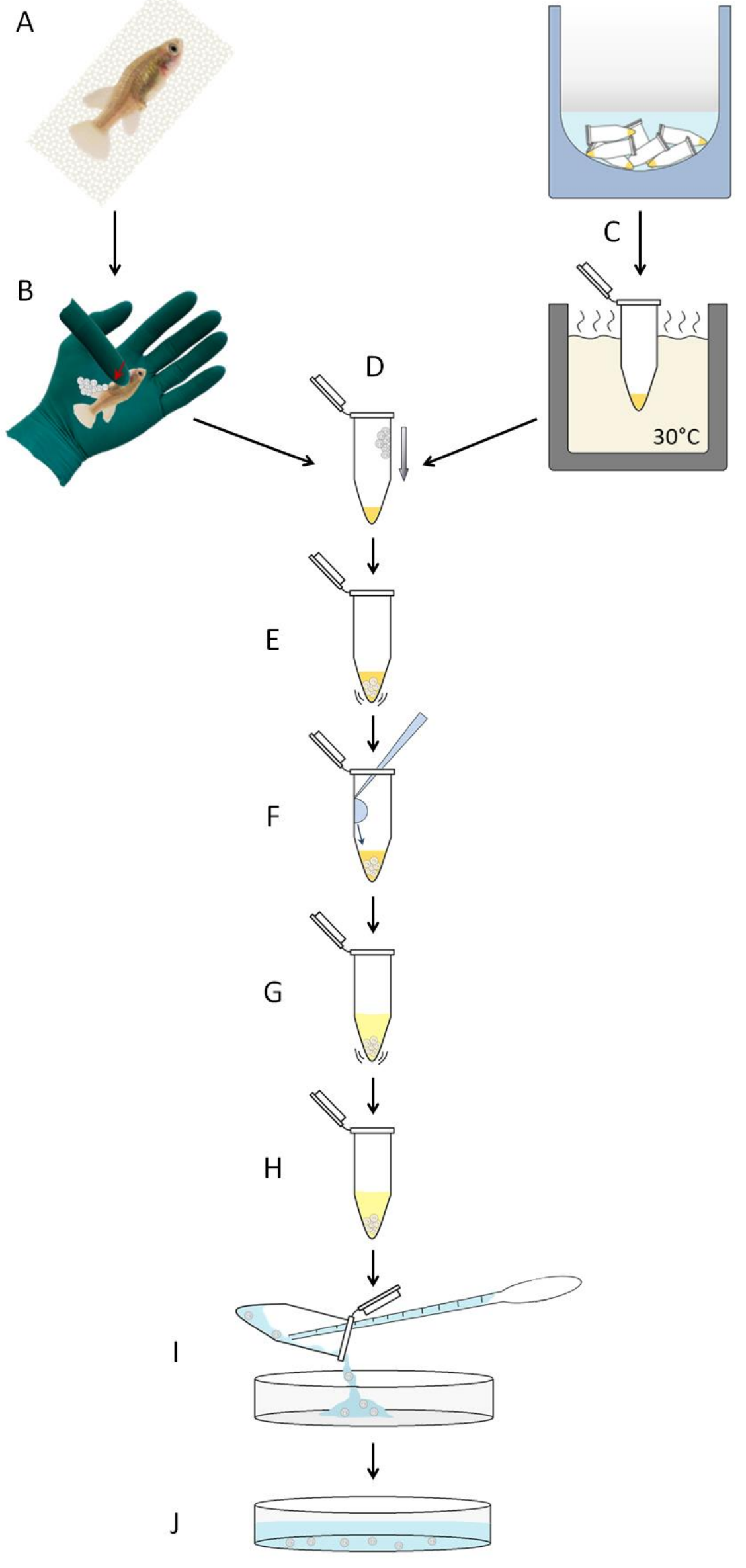


through gentle abdominal squeezing. (C) Frozen sperm thawing in a water bath. (D-E) Eggs mixed with sperm-

258 Fertilized eggs developing in a petri dish. For detailed explanation, refer to the protocol.

259 The sperm obtained from the male gonads was mixed with FBS or BSMIS buffer 260 containing $10 \%$ DMSO. Half of the sperm-extender-cryoprotectant mixture was 261 directly activated (with 1:2 or 1:4 BSMIS) and used for the fertilization of the eggs 262 (Figure 5D-J) while the other half was frozen and cryopreserved (Figure 3). A 263 subset of the sperm was also frozen in Cryovials with the same shape of the 264 1,5mL Eppendorf tubes.

265 After 2 weeks the frozen sperm were thawed, activated and used to fertilize 266 another pool of eggs derived from 10 week old females, following the same 267 procedure (Figure 5). Sperm concentrations used for fertilization were on 268 average $60,000 / \mathrm{ul}$, a value estimated by counting the number of sperm in a $3 \times 3$ 269 square field Burker chamber. Eggs fertilized in both ways were monitored until 270 the stage of mid-somitogenesis (Figure 6A) or later (Figure 6B). Survival rates 271 were scored (Figure 6F). This experiment was repeated again after 4 months 272 using aliquots from our collection. Yields of fertilization achieved were 273 comparable to those after 2 weeks freezing (Figure S1). 

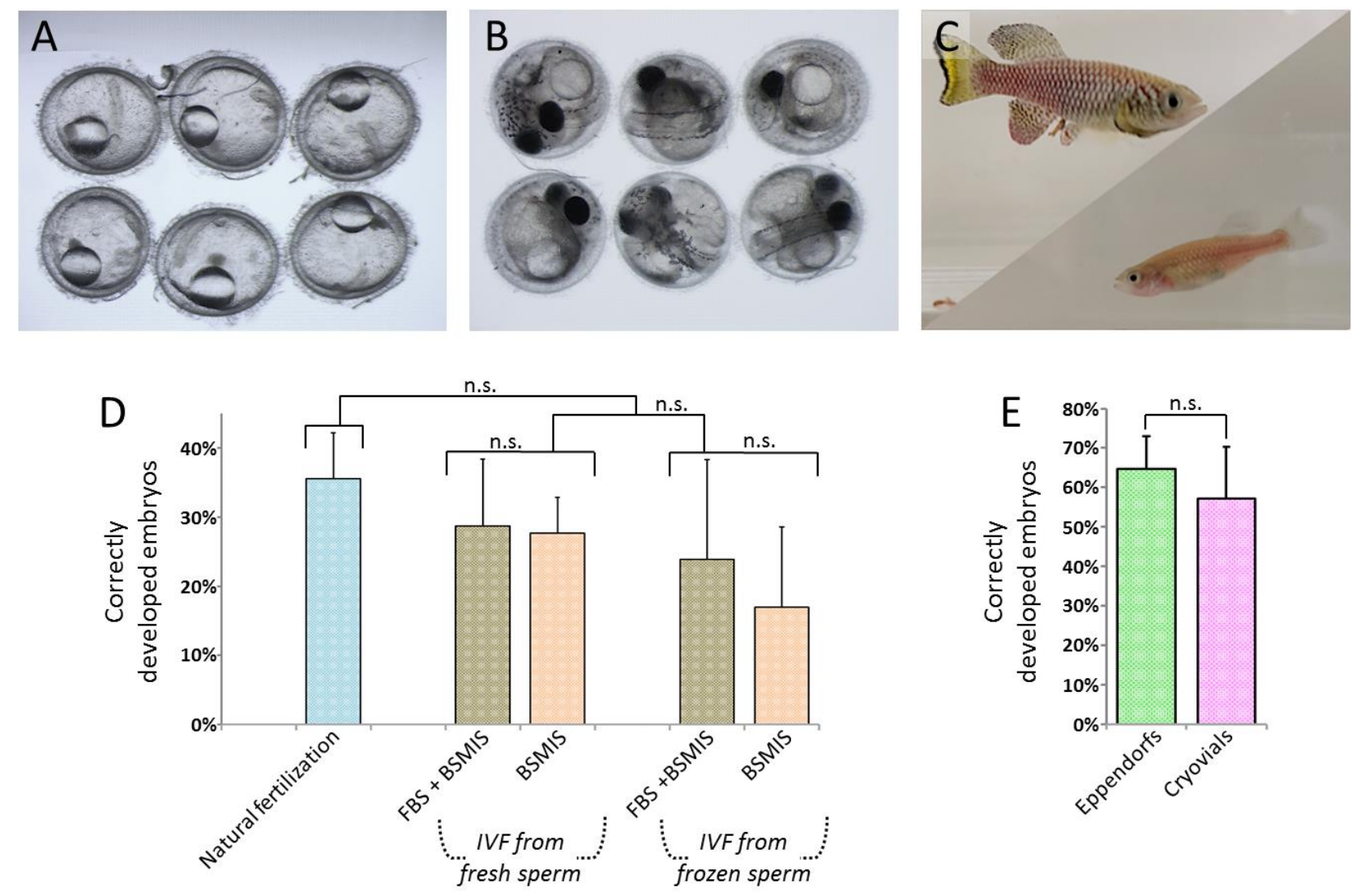

275 Figure 6: Fertilization rates and embryonic development. (A) Embryos fertilized with IVF using frozen sperm develop

276 through diapause II/mid-somitogenesis stage and later (B), up to advanced developmental stage. (C) Adult fish derived from IVF embryos. (D) Fertilization efficiencies of natural breeding, IVF with fresh extracted sperm and IVF with sperm frozen for 2 weeks. None of the condition tested resulted in fertilization rates significantly different from natural fertilization when tested with one way Anova post hoc Tukey's test. $N=6,8,4,10,3$ independent fertilization trials for each fertilization condition, respectively, in order of appearance in the graph. $N=1023,440,349,485,201$ total eggs used for fertilization in the trials for each fertilization condition, respectively, in order of appearance in the graph. (E) Fertilization efficiencies of IVF using sperm frozen for 50 days in Cryovials or Eppendorf tubes. The egg pool used for fertilization derived from 10 weeks old females. 3 independent trials were performed using different females and sperm aliquots. $\mathrm{N}=45$ total eggs used for cryovials (fertilized with 3 different sperm aliquots) IVF and 52 total eggs for Eppendorfs (fertilized with 3 different sperm aliquots) tests. T-Test between the two groups results was not significant. 
288 To approximate the fertility rate, we counted the total number of embryos that 289 developed until mid-somitogenesis (Figure 6A) over the total initial number of 290 eggs used for fertilization (Figure 6D). For the IVF experiments, several embryos 291 were followed through development to post-somitogenesis (Figure 6B), post292 hatching and up to adulthood (Figure 6C). The growth rates were normal, 293 reaching maturity in 5-6 weeks, and no developmental defects were detected $294(n=20)$. These fish were fertile and able to produce viable embryos $(n=70)$. We 295 found that under our conditions, rates of fertilization with frozen sperm was 296 approximately $25 \%$ on average and were only slightly below fresh IVF or natural 297 fertilization.

298 In a final experiment we used the sperm previously extracted and frozen in 299 Cryovials and Eppendorf tubes for 50 days to fertilize eggs of 10 weeks old 300 females. In this experiment we used FBS as extender and BSMIS diluted 1:4 in 301 deionized water as activator. Results were comparable between the two groups 302 (Figure 6E), suggesting that both containers are suitable for short-medium term 303 sperm storage. The long term storage (years) remains unexplored.

304 A detailed protocol for the entire procedure of egg in vitro fertilization is found in 305 the Supplemental Methods.

\section{Cortical reaction}

Interestingly, we noticed that upon contact with the aqueous medium, eggs could spontaneously undergo the cortical reaction even in the absence of 309 sperm, as indicated by an increase in the distance $(\lambda)$ of about $52.7 \pm 10.9 \mu \mathrm{m}$ 310 between the yolk and chorion membrane (Figure 7A, 7B). 

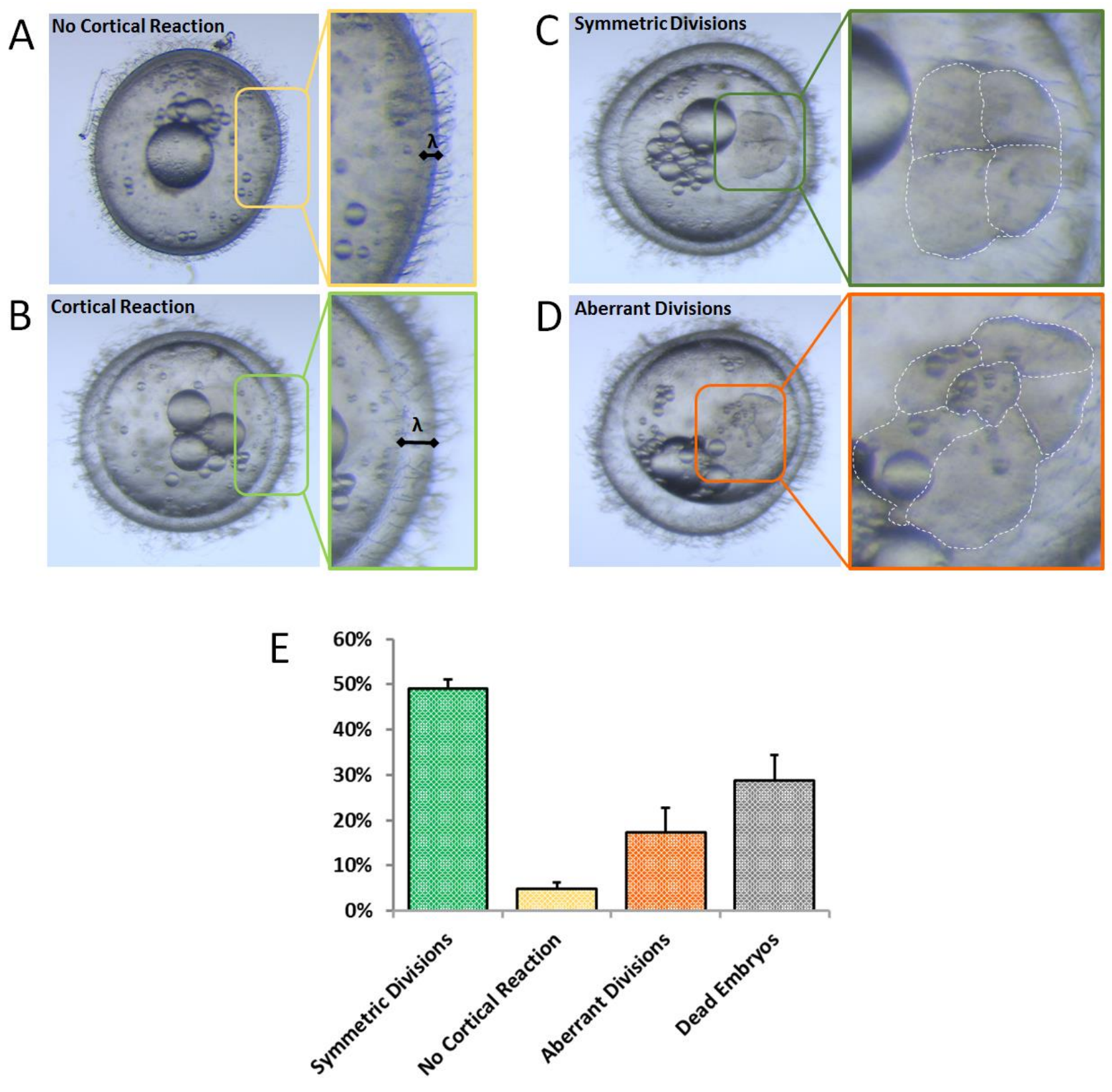

312 Figure 7: Cortical reaction and cell division in fertilized and unfertilized eggs. (A) Oocytes that do not undergo the 313 cortical display closely apposed chorion membrane and yolk ( $\lambda$ represent the average distance of $35.0 \pm 10.6 \mu \mathrm{m} . \mathrm{N}=$

8). (B) Embryo in which the cortical reaction has occurred properly where the yolk detaches from the chorion

membrane $(\lambda$ represent the average distance of $87.6 \pm 11.2 \mu \mathrm{m} . \mathrm{N}=14)$. (C) Embryo undergoing correct cell division at the 4 cell stage. (D) Pseudoembryo undergoing aberrant divisions, due to missing or incorrect fertilization. Cells are odd in number and of irregular size and shape during the earliest stages. (E) Average embryonic phenotypic distribution. $N=4$ independent natural fertilizations with 470, 332, 101, 612 eggs collected, respectively. Embryos were screened after 6 hours from collection and phenotype was assessed by eye. The graph represents the average of the 4 different collections. 
322 Under conditions of natural fertilization, a fraction (10\% to $25 \%$ ) of eggs 323 underwent spontaneous cortical reaction, while a smaller (1\%-10\%) fraction of 324 eggs remained blocked in the pre-cortical reaction stage (Figure 7E, yellow bar). 325 Eggs that underwent a spontaneous cortical reaction, which we term 326 pseudoembryos, displayed abnormal cell cleavages (compare Figure 7D and 327 7C), errant development and embryonic death in the first 10 days (Figure 7E, 328 orange bar). Eggs that did not carry out the cortical reaction were not fertilized, 329 but evidently not all eggs that carried out the cortical reaction were fertilized 330 either. Thus the cortical reaction itself does not necessarily indicate fertilization 331 success.

\section{Discussion}

In this study, we worked out conditions for sperm activation,

336 cryopreservation and in vitro fertilization in the species $N$. furzeri, aiming to

337 establish protocols to facilitate line maintenance and space usage of this fish.

338 Previous publications show how a decrease in osmolality together with a species

339 specific salt combination are required for fish sperm activation [13-14].

340 Concentrating on the most used buffers and testing them in several dilutions and

341 combination, we found that $N$. furzeri requires a specific protocol tailored for this 342 species. 
After exploring several combinations, we narrowed down the viable extender solutions to FBS and BSMIS and activators to BSMIS 1:4 and 1:2.

Cryoprotectants, 10\% DMSO and MetOH best prevented cryodamage and

preserved sperm viability. Moderate freezing rates in a beaker using dry ice, and rapid thawing at $30^{\circ} \mathrm{C}$ resulted in sperm that could be readily activated. Our IVF method resulted in fertilization rates that were comparable between frozen sperm, fresh sperm and natural fertilization, hovering around $20-30 \%$.

Cryopreserved sperm was equally active at least 4 months later.

Nevertheless, there are several parameter that we did not explore and possible limitations of our study. First and foremost, we do not know the maximal length of time that sperm can remain well preserved under these conditions.

Based on other reports ${ }^{28}$, we anticipate preservation could last many years. We used sperm densities of ca. $60,000 / \mu \mathrm{l}$ and male age of 9-11 weeks; we did not systematically optimize these parameters. Another limitation of our study is that males were sacrificed in order to retrieve sperm from the testes. Future techniques could be developed to collect sufficient sperm from live animals. Finally, sperm preservation only represents solving half the problem. In the 361 as well.

Currently most $N$. furzeri lines are kept in embryonic diapause for longterm maintenance. This still remains a very useful approach because it preserves lines in the form of organism, not as gametes, and does not require freezers or liquid nitrogen systems that can breakdown. However, it still remains unclear how long embryos can be maintained in diapause, but it is likely less time than cryopreservation of sperm. Maintaining embryos in diapause is more 
368 time consuming and takes up greater space. Whether animals in embryonic

369 diapause can also be cryopreserved is also unknown. In any case, sperm

370 cryopreservation techniques and IVF can complement existing techniques of

371 diapause preservation.

$372 \quad$ One of the unexpected observations we made in the course of our studies

373 is that a considerable number of oocytes undergo the cortical reaction even in 374 the absence of sperm, with either IVF or natural fertilization. Such 375 "pseudoembryos" displayed abnormal development. Though the mechanism 376 underlying spontaneous cortical reaction remains unknown in this species, it is 377 thought to be related to the degree of egg maturation ${ }^{29,30}$. Whether conditions 378 can be improved to minimize such events to increase the fraction of fertilizable 379 oocytes is another parameter that deserves future attention.

\section{Conclusion}

381 In sum, we worked out conditions feasible for sperm cryopreservation and in vitro 382 fertilization in Nothobranchius furzeri. Our protocol provides useful techniques 383 that can enhance existing approaches for line maintenance and husbandry, 384 which should greatly facilitate the use of Nothobranchius furzeri as a model 385 organism. 


\section{Materials and Methods}

\section{Fish husbandry and sample collection}

392 All adult fish used were Nothobranchius furzeri belonging to the GRZ strain and were raised singularly in $2.8 \mathrm{~L}$ tanks from the second week of life. Water parameters were $\mathrm{pH}: 7-8 ; \mathrm{Kh}: 3-5 ; \mathrm{T}: 27^{\circ} \mathrm{C} .10 \%$ of the water in the system was automatically replaced with clean water every day. Fish were raised in 12 hours of light and 12 hours of darkness. Fish were fed with chironomus twice a day and

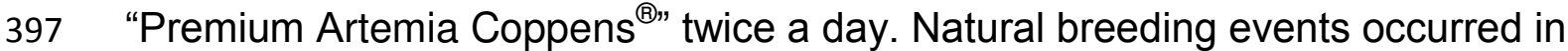
398 8L tanks with 1 male and 3-5 females. To initiate breeding, one box $(9 \mathrm{~cm} \times 9 \mathrm{~cm}$ $x 4 \mathrm{~cm})$ half full of river sand $(\varnothing<0,2 \mathrm{~mm})$ was put on the bottom of the tank. The boxes were put in the fish tanks for 2-3 hours during which the average number

401 of eggs laid by each female in the sand was between $5-30$. All the fish used for 402 the experiments were from 9-11 weeks old.

\section{Ethical approval}

404 All fish husbandry was performed in the fish facility of the Max Planck Institute for 405 Biology of Aging (Permit Nr. 576.1.36.6.G12/18 Be). The animal experiments 406 were approved by the Institutional Animal Welfare Body (RB.16.005) and 407 complied with national legislation and the European directive 2010/63/EU. The 408 study was overseen and reported to the appropriate authority, the Veterinarian 409 State Office Cologne.

\section{Gonad extraction and sperm collection}

412 Males were anesthetized with tricaine methanesulfonate (Sigma, $0.5 \mathrm{mg} / \mathrm{ml}$ ) for $41310+$ minutes, until movement and breathing ceased. Fish were dried using tissue 
414 paper and decapitated. The abdomen was cut open with scissors, the organs 415 removed and the testes flanking the swimming bladder were gently extracted 416 using a forceps. Gonads were placed in an Eppendorf tube containing $500 \mu \mathrm{l}$ of 417 extender solution, held with forceps and spun back and forth for $1 \mathrm{~min}$. After the 418 sperm was released into the solution, the gonads were removed from the 419 Eppendorf tube. On average around 30 million sperm (between 7,5 million and 420100 million) were released. This value was estimated by counting the sperm cells 421 of several sperm-extender aliquots in a $3 \times 3$ square field of a Burker chamber. 422 The initial total sperm value was calculated multiplying the number obtained by 423 the dilution factor.

\section{Fresh solutions trials (Figure 1)}

425 Each individual gonad was placed in $250 \mu$ l of solution (such as tank water, DE 426 water, BSMIS, HBSS, FBS, Iwamatsu solution) at dilutions of $(1: 1,1: 2,1: 4,1: 8)$ 427 and the sperm was released inside by shaking for $5-10$ seconds. $10 \mu$ of each 428 solution+sperm mix were placed in a hemocytometer chamber immediately after 429 the activation of sperm, visualized and recorded. Each trial combination was 430 repeated at least 3 times with gonads derived from different fish.

431 BSMIS: (75mM NaCl, $70 \mathrm{mM} \mathrm{KCl}^{2} 2 \mathrm{mM} \mathrm{CaCL}_{2}, 1 \mathrm{mM} \mathrm{MgSO}_{4}, 20 \mathrm{mM}$ Tris pH8.0)

432 HBSS: (Gibco $\left.{ }^{\mathrm{TM}} \# 14025-092\right)$

433 FBS: (Gibco® \#10270-106)

434 Extender solution sperm activation trials (Figure 2)

435 One gonad was extracted and shaken in extender solutions as described above. 436 One volume $(3 \mu \mathrm{l})$ of sperm-extender mixture was mixed with two $(6 \mu \mathrm{l})$ or nine 
437 volumes $(27 \mu \mathrm{l})$ of activator solutions and immediately transferred to the 438 hemocytometer chamber under the microscope for video recording. Each trial 439 combination was repeated at least 3 times with gonads derived from different fish 440 shaken in the extender.

441 Imaging and video acquisition

442 For sperm visualization, we used a Zeiss Imager Z1 microscope and recorded 443 videos with a Zeiss Axiocam 506 mono, using parameters of binning $4443 \times 3$,resolution $2752 \times 2208$ pixels, and the ZEN 2.3 Pro software. Each video was 445 recorded for 8 seconds.

446 Imaris conversion and sperm motility analysis.

447 Videos were imported in Imaris 8.1 and frame time points rescaled by 10 times. 448 Black and white colors were inverted in order to visualize white dots over a black 449 background. The particle tracking function was set to identify all sperm with a 450 diameter of $\pm 2.9 \mu \mathrm{m}$. Movements were tracked with the autoregressive motion 451 algorithm function, set with maximum distance of $25 \mu \mathrm{m}$ and a maximum gap 452 size of 5 . Statistics relative to track displacement length were exported as excel 453 file and analyzed. As a cutoff, we set a displacement length of $>15 \mu \mathrm{m}$ to 454 distinguish directionally traveling sperm from non-active or vibrating sperm. In 455 case of general drift of the whole sample size we increased the cutoff to $>35 \mu \mathrm{m}$ 456 or $>50 \mu \mathrm{m}$ (in case of very strong drift).

\section{Cryoprotectant trials (Figure 4A,B)}

458 For each biological replicate the two testes were extracted from a male and were 459 shaken into $500 \mu$ l of different extender solutions (FBS or BSMIS). In total we 460 used 3 biological replicates for each extender combination. Mixes were aliquoted 
461 in smaller volumes $(60 \mu \mathrm{l})$ and different cryoprotectants (DMSO (Sigma), DMF 462 (Carl Roth), Methanol (Optima), DMA (Sigma) or glycerol $\geq 99.5 \%$ (Sigma) were 463 added in different concentrations in each Eppendorf and immediately mixed. $3 \mu \mathrm{l}$ 464 from each sample were taken and mixed with an activation solution to check the 465 sperm mobility, following the procedure previously described. The rest of each 466 mix was frozen following the cryopreservation procedure. Sperm survival was 467 checked after two weeks upon thawing (see below).

468 Cryopreservation (Figure 5)

469 Extender-sperm-cryoprotectant mixtures were incubated in $1.5 \mathrm{ml}$ Eppendorf 470 tubes for 1 hour at $4^{\circ} \mathrm{C}$. The mixtures were distributed in Eppendorf tubes at a 471 volume of $60 \mu \mathrm{l}$ each, the tubes were closed and gently placed at the bottom of a 472 glass beaker sitting in a styrofoam box filled up to $10 \mathrm{~cm}$ with dry ice. After 15 473 minutes, Eppendorf tubes were quickly moved into a rack in a nitrogen tank, 474 exposed to nitrogen vapors for 30 minutes. Samples were moved directly into 475 liquid nitrogen for long term storage.

\section{$476 \quad$ Freezing trials (Figure 4C)}

477 Both gonads from the same individual were placed in $500 \mu$ FBS solution and 478 swung for sperm release as previously described. $60 \mu$ of the resulting mix were 479 mixed half with 10\% DMSO and half with 10\% methanol in Eppendorf tubes (or 480 Cryovials, Sarstedt \#72.692.005) and incubated for 1 hour at $4^{\circ} \mathrm{C}$. Vials were 481 distributed to $\mathrm{Mr}$. frosty container $\left(-10^{\circ} \mathrm{C}\right.$ per min), a beaker surrounded with dry 482 ice $\left(-20^{\circ} \mathrm{C}\right.$ per min), directly on dry ice $\left(-50^{\circ} \mathrm{C}\right.$ per min), directly in nitrogen gas 483 phase $\left(-100^{\circ} \mathrm{C}\right.$ per min), directly in nitrogen liquid $\left(-200^{\circ} \mathrm{C}\right.$ per min) phase for 30 484 minutes, until completely frozen. The samples were placed in the nitrogen liquid 
485 phase after they reached a temperature of below $-50^{\circ} \mathrm{C}$. The thawing and 486 monitoring process were performed as described in the main text.

487 Thawing and sperm revival (Figure 4 D)

488 Frozen samples were thawed directly from liquid nitrogen into a $30^{\circ} \mathrm{C}$ warm 489 water bath until the ice completely melted. The procedure usually took 1 minute 490 or less. One volume $(3 \mu \mathrm{l})$ of thawed sperm mixtures was mixed with two volumes $491(6 \mu \mathrm{l})$ of BSMIS 1:4 and pipetted into a hemocytometer chamber to record sperm 492 movements.

\section{In vitro fertilization by thawed sperm (Figure 5,6)}

494 Females were anesthetized in a Tricaine methanesulfonate solution $(0.5 \mathrm{mg} / \mathrm{ml})$ 495 and carefully dried of residual water to prevent spontaneous egg cortical reaction 496 or activation (Figure 5A). Placing the fish on an open hand, a gentle pressure 497 was applied with a finger on the abdomen, pushing gently from the middle 498 toward the anus (Figure $5 \mathrm{~B}$ ). Typically 5 to 35 eggs were expelled. Eggs were 499 collected using a forceps into an Eppendorf tube containing the extender-sperm 500 solution (Figure 5D). In the case of frozen sperm, the tube was thawed 501 immediately before fertilization in a $30^{\circ} \mathrm{C}$ water bath (Figure $5 \mathrm{C}$ ). These steps 502 were best performed by two people, with one person thawing the sperm while 503 the other person expelled eggs from the female. Eggs were placed at the edge of 504 the tube and gently pushed to the bottom (Figure 5D). We used aliquots of 505 sperm-extender of $60 \mu \mathrm{l}$ and no more than 35 eggs per aliquot. Once the eggs 506 were immersed, the tube was gently flicked for 10-20 seconds (Figure 5E), 507 allowing homogeneous distribution of sperm around the eggs. The activator 508 solution was then pipetted into the tube, letting drops slide over the tube's border 
509 (Figure 5F) and then mixed with the extender by gently flicking the tube for 20-30 510 seconds (Figure 5G). The activated sperm was incubated with the eggs for 10 511 minutes with the tubes standing open on a bench at room temperature (Figure $5125 \mathrm{H}$ ). At this step, $10 \mathrm{ul}$ of the mixture was examined under the microscope to 513 evaluate sperm motility. To avoid damage due to DMSO toxicity, the embryos 514 were transferred after 10 minutes by pipette to a petri dish and methylene blue 515 buffered tank water (Figure 5I). The water was replaced twice and the petri dish 516 incubated at $28^{\circ} \mathrm{C}$ (Figure 5J).

519 Embryo phenotype assessment and cortical reaction. (Figure 7)

52010 breeding tanks were set up with 1 male and 4 females each. The fish were 521 allowed to breed for 3-4 hours and then eggs were collected and cleaned of 522 empty shells or dead eggs and allowed to develop in an incubator at $28^{\circ} \mathrm{C}$ for 6 523 hours. Embryos were then carefully examined under a Leica M80 524 stereomicroscope to assess morphology, cortical reaction, cell shape and 525 number. Four independent breedings were performed with at least 2 days 526 between them. After 6 hours in the incubator, each breeding event yielded 470 , $527332,101,612$ embryos, respectively. Data regarding the percentages of the various 528 phenotypes were averaged between the 4 collections to produce the graph.

\section{Graph production images acquisition and enhancement}

530 Raw data depicting track displacement length realized with Imaris were exported 531 as an excel chart. Data relative to all the experiments were pooled in a unique 532 excel file, containing 3 replicates for each individual trial or combination. Total 
533 data were used to calculate averages, standard deviations and standard errors, 534 in case of mean of the means. Graphs were produced from these data using 535 excel.

536 Tracking images were acquired from videos using Imaris snapshot function.

537 Brightfield images were acquired using a Leica M80 microscope equipped with a 538 Leica MC170 HD camera. Images were enhanced in brightness, contrast and 539 saturation using GIMP to improve the visual quality. Quantifications were not 540 affected by imaging quality enhancing modifications.

541 Graphics and drawings were realized using Paint, GIMP and Power point.

543 Data availability

544 All data generated or analysed during this study are included in this 545 published article (and its Supplementary Information files).

546 The datasets generated during and/or analysed during the current study 547 are available from the corresponding author on reasonable request.

\section{References}

549 1. Genade, T. et al. Annual fishes of the genus Nothobarnchius as a model system for $550 \quad$ aging research. Aging Cell (2005) doi:10.1111/j.1474-9726.2005.00165.x.

551 2. Cellerino, A., Valenzano, D. R. \& Reichard, M. From the bush to the bench: The annual Nothobranchius fishes as a new model system in biology. Biol. Rev. (2016) doi:10.1111/brv.12183. 
554 3. Harel, I. et al. A platform for rapid exploration of aging and diseases in a naturally short-lived vertebrate. Cell (2015) doi:10.1016/j.cell.2015.01.038.

556 4. Hedrick, P. W. Inbreeding depression in conservation biology. Annu. Rev. Ecol. Syst. 557 (2000) doi:10.1146/annurev.ecolsys.31.1.139.

558 5. Wright, L. I., Tregenza, T. \& Hosken, D. J. Inbreeding, inbreeding depression and 559

9. Kanuga, M. K. et al. Effect of aging on male reproduction in zebrafish (Danio rerio). J. Exp. Zool. Part A Ecol. Genet. Physiol. (2011) doi:10.1002/jez.661.

10. Valenzano, D. R., Sharp, S. \& Brunet, A. Transposon-mediated transgenesis in the short-lived african killifish nothobranchius furzeri, a vertebrate model for aging. G3 Genes, Genomes, Genet. (2011) doi:10.1534/g3.111.001271.

12. Romney, A. L. T., Davis, E. M., Corona, M. M., Wagner, J. T. \& Podrabsky, J. E. 
Temperature-dependent Vitamin D signaling regulates developmental trajectory associated with diapause in an annual killifish. Proc. Natl. Acad. Sci. U. S. A. (2018) doi:10.1073/pnas.1804590115.

13. Hu, C. K. \& Brunet, A. The African turquoise killifish: A research organism to study vertebrate aging and diapause. Aging Cell (2018) doi:10.1111/acel.12757.

14. Yang, H. \& Tiersch, T. R. Current status of sperm cryopreservation in biomedical research fish models: Zebrafish, medaka, and Xiphophorus. Comparative Biochemistry and Physiology - C Toxicology and Pharmacology (2009) doi:10.1016/j.cbpc.2008.07.005.

15. Cabrita, E. et al. Cryopreservation of fish sperm: Applications and perspectives. Journal of Applied Ichthyology (2010) doi:10.1111/j.1439-0426.2010.01556.x.

16. Yang, H. \& Tiersch, T. R. Sperm motility initiation and duration in a euryhaline fish, medaka (Oryzias latipes). Theriogenology (2009) doi:10.1016/j.theriogenology.2009.03.007.

17. Morisawa, M. \& Suzuki, K. Osmolality and potassium ion: Their roles in initiation of sperm motility in teleosts. Science (80-. ). (1980) doi:10.1126/science.7444445.

18. Pérez, L. et al. Role of calcium on the initiation of sperm motility in the European eel. Comp. Biochem. Physiol. -Part A Mol. Integr. Physiol. (2016) doi:10.1016/j.cbpa.2015.10.009.

19. Stoss, J. \& Holtz, W. Cryopreservation of rainbow trout (Salmo gairdneri) sperm. II. Effect of $\mathrm{pH}$ and presence of a buffer in the diluent. Aquaculture (1981) doi:10.1016/0044-8486(81)90183-6. 
20. Morita, M., Takemura, A. \& Okuno, M. Requirement of $\mathrm{Ca} 2+$ on activation of sperm motility in euryhaline tilapia Oreochromis mossambicus. J. Exp. Biol. (2003) doi:10.1242/jeb.00153.

21. Aoki, K., Okamoto, M., Tatsumi, K. \& Ishikawa, Y. Cryopreservation of Medaka Spermatozoa. Zoolog. Sci. (1997) doi:10.2108/zsj.14.641.

22. Beirão, J., Lewis, J. A., Wringe, B. F. \& Purchase, C. F. A novel sperm adaptation to evolutionary constraints on reproduction: Pre-ejaculatory sperm activation in the beach spawning capelin (Osmeridae). Ecol. Evol. (2018) doi:10.1002/ece3.3783.

23. Ohta, H., Kawamura, K., Unuma, T. \& Takegoshi, Y. Cryopreservation of the sperm of the Japanese bitterling. J. Fish Biol. (2001) doi:10.1006/jfbi.2000.1477.

24. Dong, Q., Huang, C. \& Tiersch, T. R. Post-thaw amendment of cryopreserved sperm for use in artificial insemination of a viviparous fish, the green swordtail Xiphophorus helleri. Aquaculture (2006) doi:10.1016/j.aquaculture.2006.05.011.

25. Bhattacharya, S. Cryopretectants and Their Usage in Cryopreservation Process. in Cryopreservation Biotechnology in Biomedical and Biological Sciences (2018). doi:10.5772/intechopen.80477.

26. Yang, H., Norris, M., Winn, R. \& Tiersch, T. R. Evaluation of cryoprotectant and cooling rate for sperm cryopreservation in the euryhaline fish medaka Oryzias latipes. Cryobiology (2010) doi:10.1016/j.cryobiol.2010.07.006.

27. Gao, D. \& Critser, J. K. Mechanisms of cryoinjury in living cells. ILAR J. (2000) doi:10.1093/ilar.41.4.187.

28. Marinović, Z. et al. Preservation of zebrafish genetic resources through testis 
cryopreservation and spermatogonia transplantation. Sci. Rep. (2019) doi:10.1038/s41598-019-50169-1.

29. Gilkey, J. C. Mechanisms of fertilization in fishes. Integr. Comp. Biol. (1981) doi:10.1093/icb/21.2.359.

30. LONGO, F. J., SO, F. \& SCHUETZ, A. W. MEIOTIC MATURATION AND THE CORTICAL GRANULE REACTION IN STARFISH EGGS. Biol. Bull. (1982) doi:10.2307/1541457.

\section{Acknowledgements}

Imaging analyses were performed in the FACS \& Imaging Core Facility at the Max Planck Institute for Biology of Ageing, Cologne.

The authors thank Mattias Werres, Christina Paetzold and Nadine Hochhard for the great help with fish husbandry and general bench work.

\section{Author contributions}

LD: Conceived and designed the analysis, collected and analyzed the data, wrote the paper.

TKS: Conceived and designed the analysis, collected and analyzed the data, revised the paper.

RR: Analyzed the data, revised the paper.

AA: Conceived and designed the analysis, wrote the paper. 


\section{Competing interests}

643 The author(s) declare no competing interests.

\section{Supporting information}

645 Figure S1. Sperm resilience in FBS + 10\% DMSO extender. Fertilization efficiencies of IVF

with sperm frozen for 2 weeks or 4 months. $N=10$ independent fertilization trials ( 485 total eggs used) with sperm frozen for 2 weeks in FBS $+10 \%$ DMSO extender and $\mathrm{N}=1$ fertilization trial (52 total eggs used) with sperm frozen for 4 months in FBS $+10 \%$ DMSO extender.

S1 File. IVF protocol. This is the protocol that we suggest and that uses FBS as extender, DMSO $10 \%$ as cryoprotectant and BSMIS 1:4 as activator. 


\section{Figures}
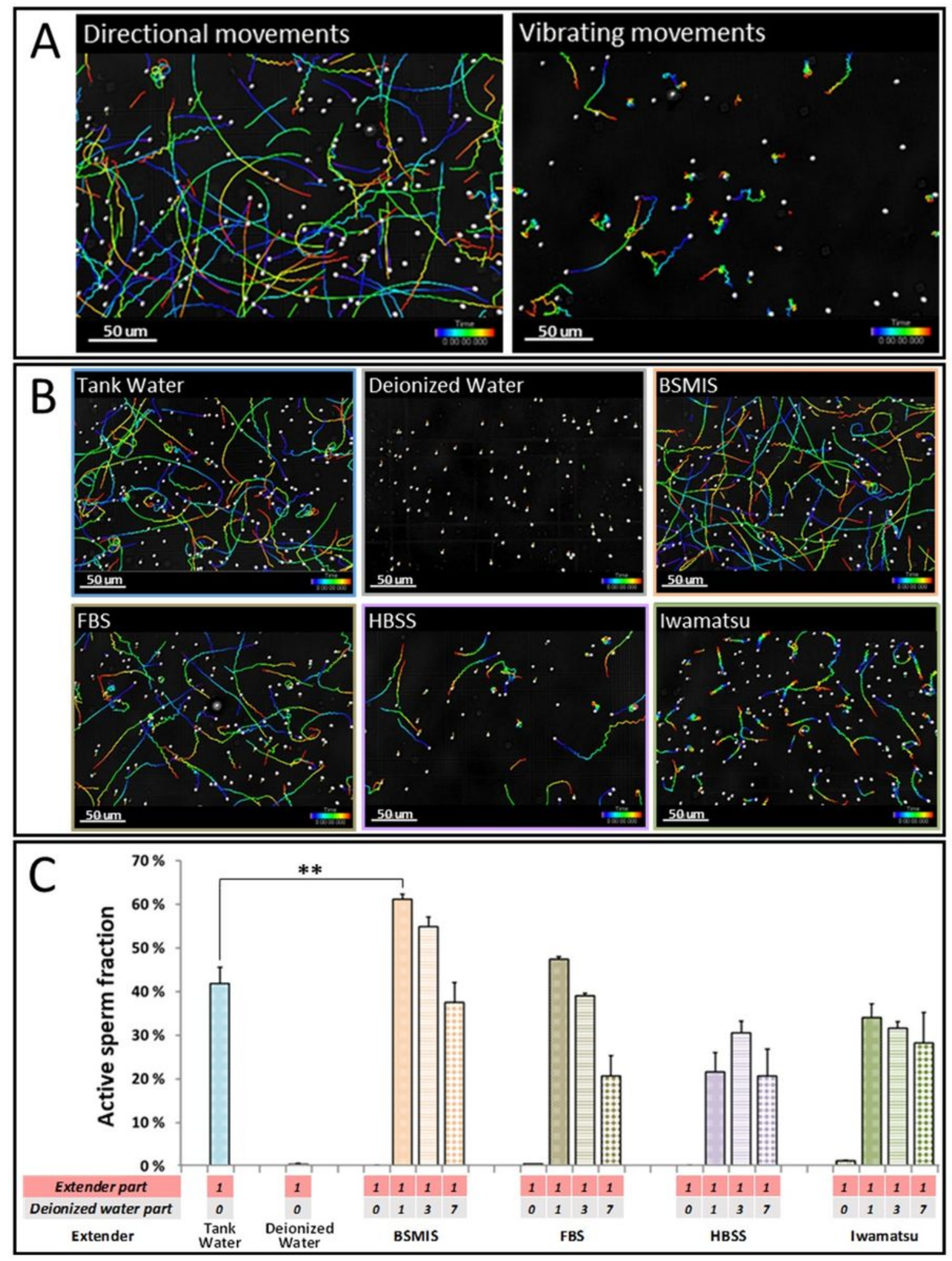

\section{Figure 1}

Fresh sperm activation in different buffers. (A) Motility tracing of different types of sperm movement that occur after activation, resulting in proper directional movements (left) or erratic inefficient vibrating movements (right). (B) Representative images of sperm movement in activated by 1:1 dilution of the 
indicated buffer conditions (C) Quantification of sperm activation in different buffers calculated as the average of number of active sperm divided by total sperm among biological replicates. Asterisks indicate solutions that are significantly more effective than tank water in activating sperm based on a two way Anova post hoc Dunnett's test. . ${ }^{\star} \mathrm{p}<0.05,{ }^{\star \star} \mathrm{p}<0.01,{ }^{\star \star *} \mathrm{p}<0.001$. $\mathrm{N}=3$ biological replicates for each condition.
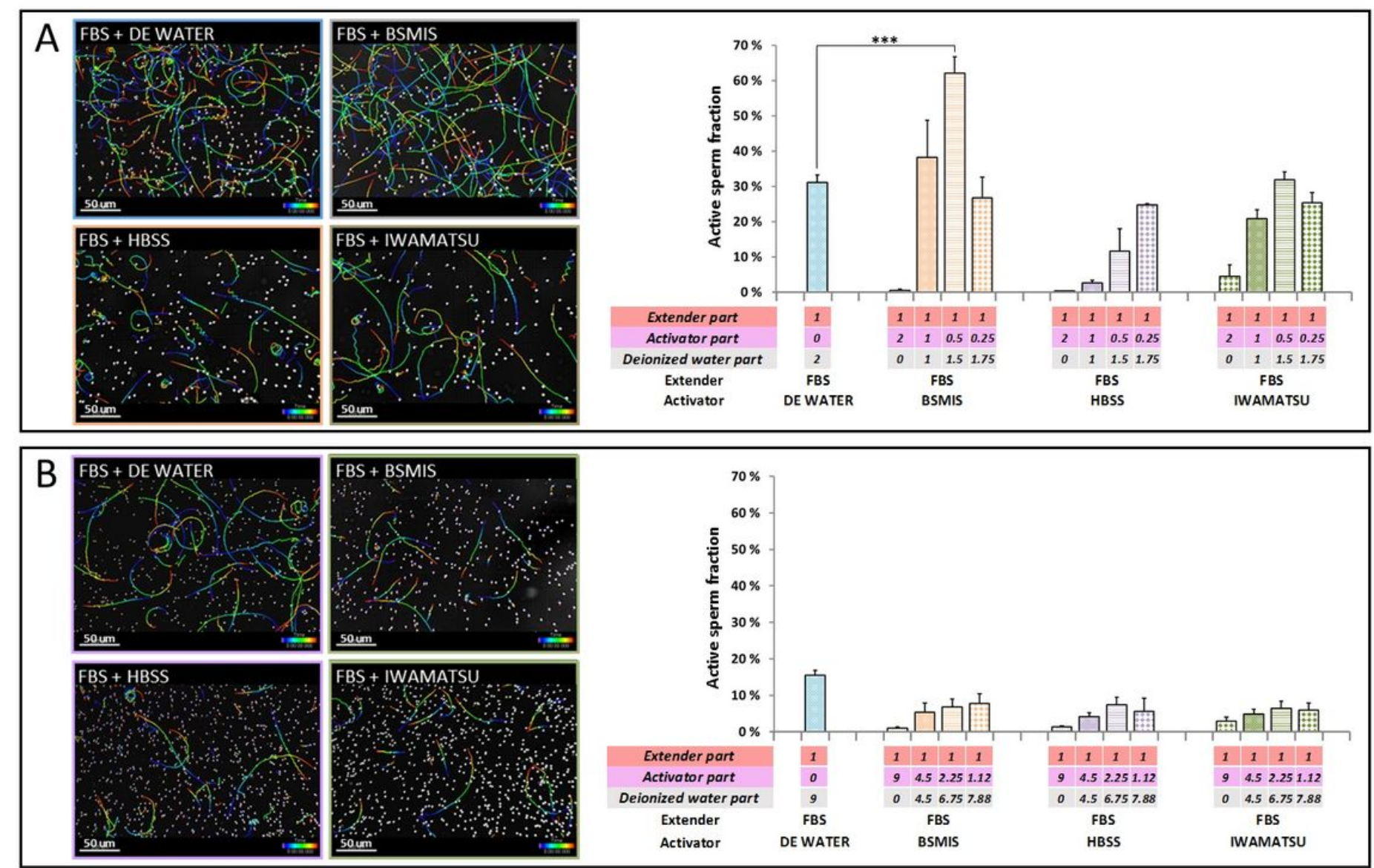

Figure 2

Fresh sperm activation using FBS as extender and different buffers for dilution. (A) Image tracking of sperm movements and quantification of directional sperm in different activating solutions, mixed in a ratio 1 to 3 with the FBS extender solution (1 volume of extender solution plus 2 volumes of activating solution at different dilutions). Ratios of extender, activator, and water for each mix are shown below each column. (B) Image tracking of sperm movements and quantification of directional sperm in different activating solutions, mixed in a ratio of 1 to 10 with the FBS extender solution (1 volume of extender solution plus 9 volumes of activating solution at different dilutions). Ratios of extender, activator, and water for each mix are shown below each column. Asterisks indicate solutions significantly more effective than tank water in activating sperm, based on a two way Anova post hoc Dunnett's test. * $\mathrm{p}<$ $0.05,{ }^{*} \mathrm{p}<0.01,{ }^{* \star} \mathrm{p}<0.001 . \mathrm{N}=3$ biological replicates for each condition. 

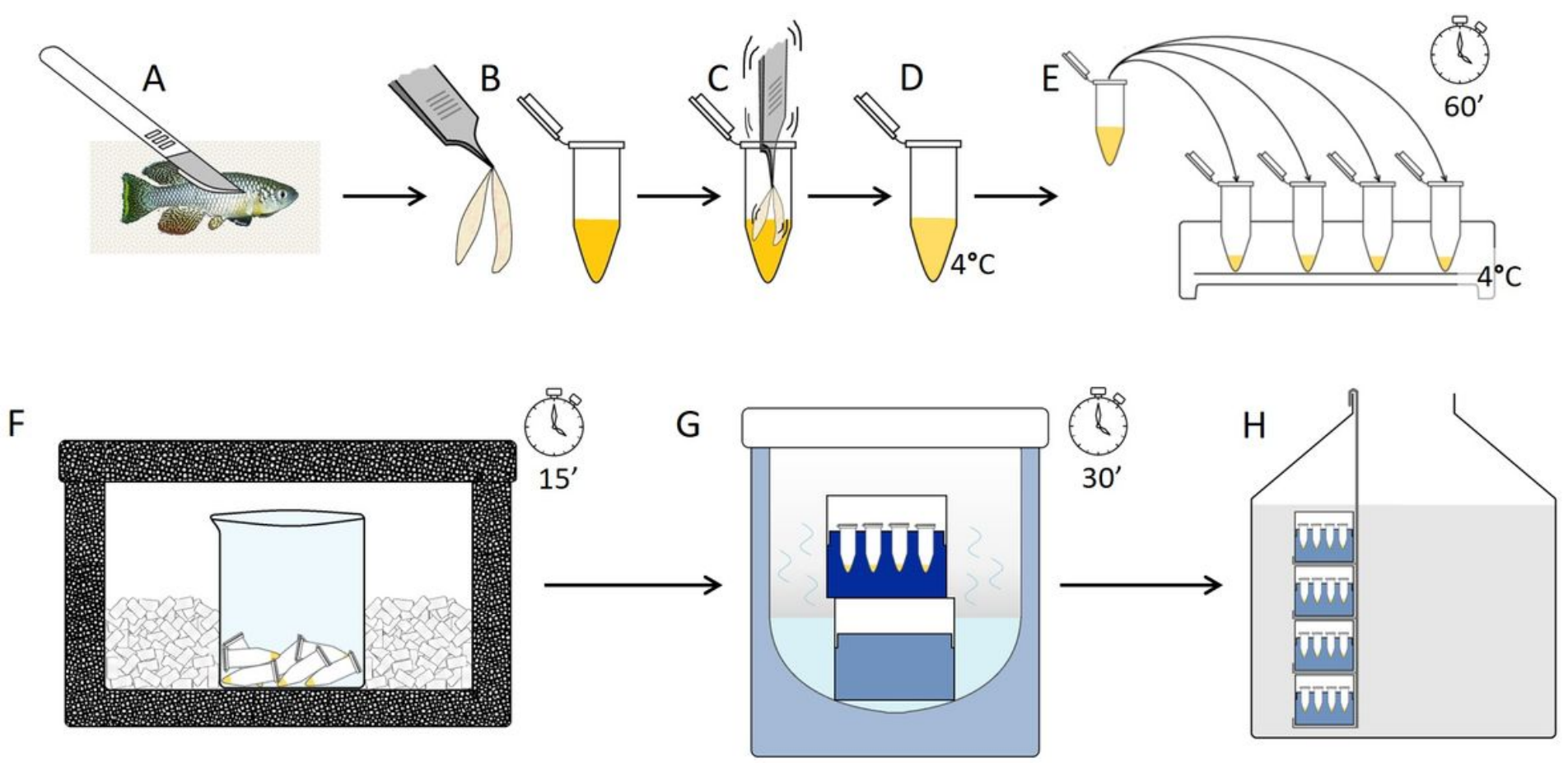

Figure 3

Schematic of sperm collection and freezing procedure. (A) Male dissection. (B) Gonad extraction. (C) Gonads are spun in extender plus cryoprotectant solution. (D) Cryoprotectant incubation with sperm cells at $4^{\circ} \mathrm{C}$. (E) Solution transfer in smaller aliquots to vials. (F) First freezing step setup with vials laying on the bottom of a glass beaker surrounded by dry ice in a closed styrofoam box. (G) Second freezing step setup with vials exposed to nitrogen vapor. $(\mathrm{H})$ Long term storage of vials in liquid nitrogen. For a detailed explanation, see text. 

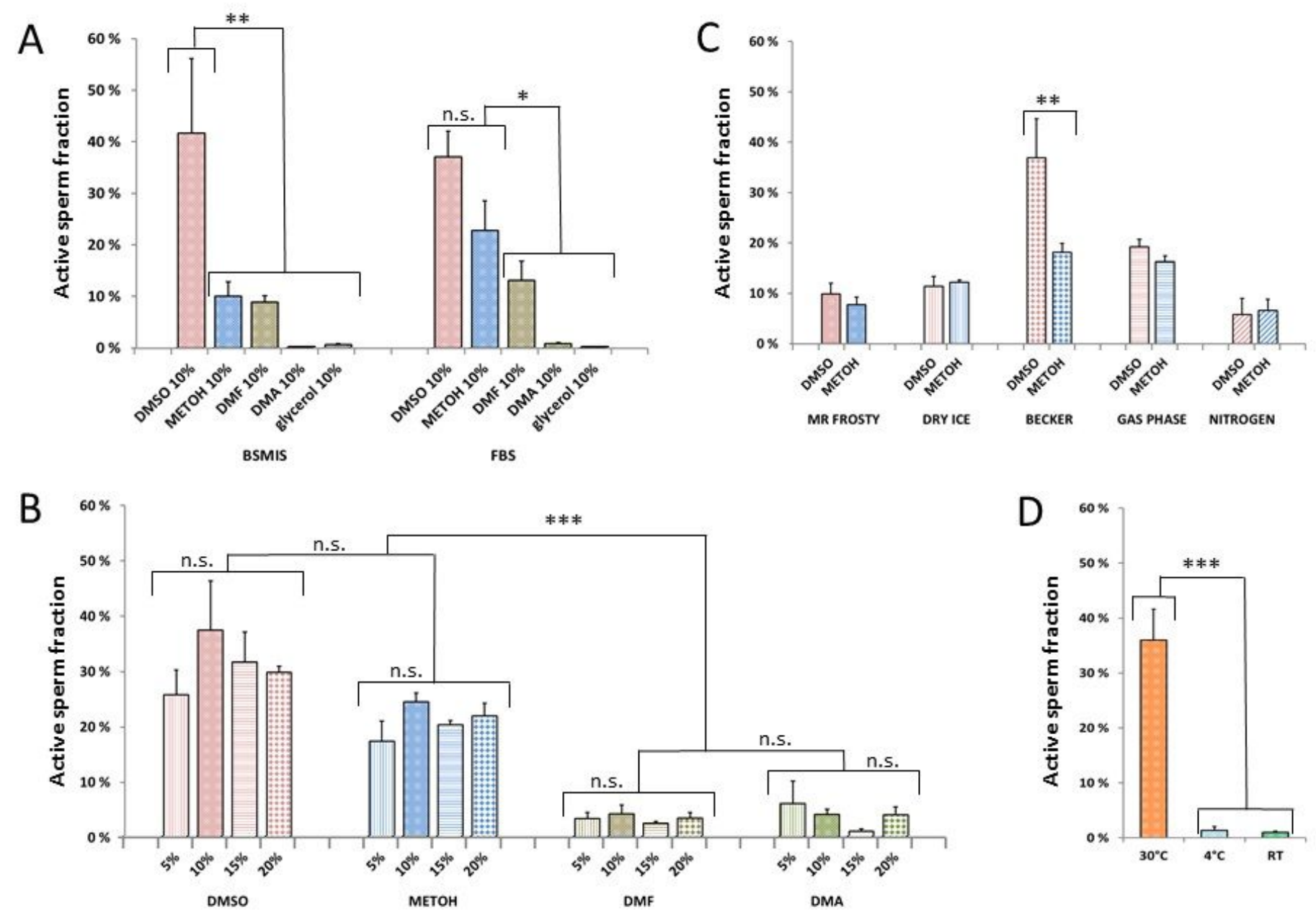

\section{Figure 4}

Cryoprotectants, cryopreservation and thawing efficiencies. (A) Effect of different cryoprotectants on sperm activation using BSMIS and FBS extender solutions. $Y$ bar represents the portion of sperm able to reactivate upon thawing. Statistical analysis used is two way Anova with multiple comparison Tukey's post hoc correction. In BSMIS extender, DMSO performed significantly better compared to the other cryoprotectants. In FBS extender, DMSO and MetOH performed significantly better compared to the other cryoprotectants.(B) Effect of different concentrations of cryoprotectants in FBS extender. Asterisks indicate significance levels of two way Anova multiple comparison test with post hoc Dunnett's correction. ${ }^{*} p<0.05,{ }^{*} p<0.01,{ }^{* \star} p<0.001$ (C) Different freezing methods with different cooling rates applied to FBS extender plus $10 \%$ cryoprotectant. Asterisks indicate significance levels of two way Anova multiple comparison test with post hoc Sidak's correction. ${ }^{\star} p<0.05,{ }^{\star \star} p<0.01$, ${ }^{\star \star *} p<0.001$ (D) Different thawing methods $\left(30^{\circ} \mathrm{C}\right.$ water bath, $4^{\circ} \mathrm{C}$ refrigerator, RT, room temperature) with different thawing rates applied to FBS extender plus 10\% DMSO. Asterisks indicate significance levels of one way Anova multiple comparison test with post hoc Tukey's correction. ${ }^{*} \mathrm{p}<0.05$, ${ }^{* \star} \mathrm{p}<0.01,{ }^{\star \star \star} \mathrm{p}<0.001 . \mathrm{N}=3$ biological replicates for each condition. 


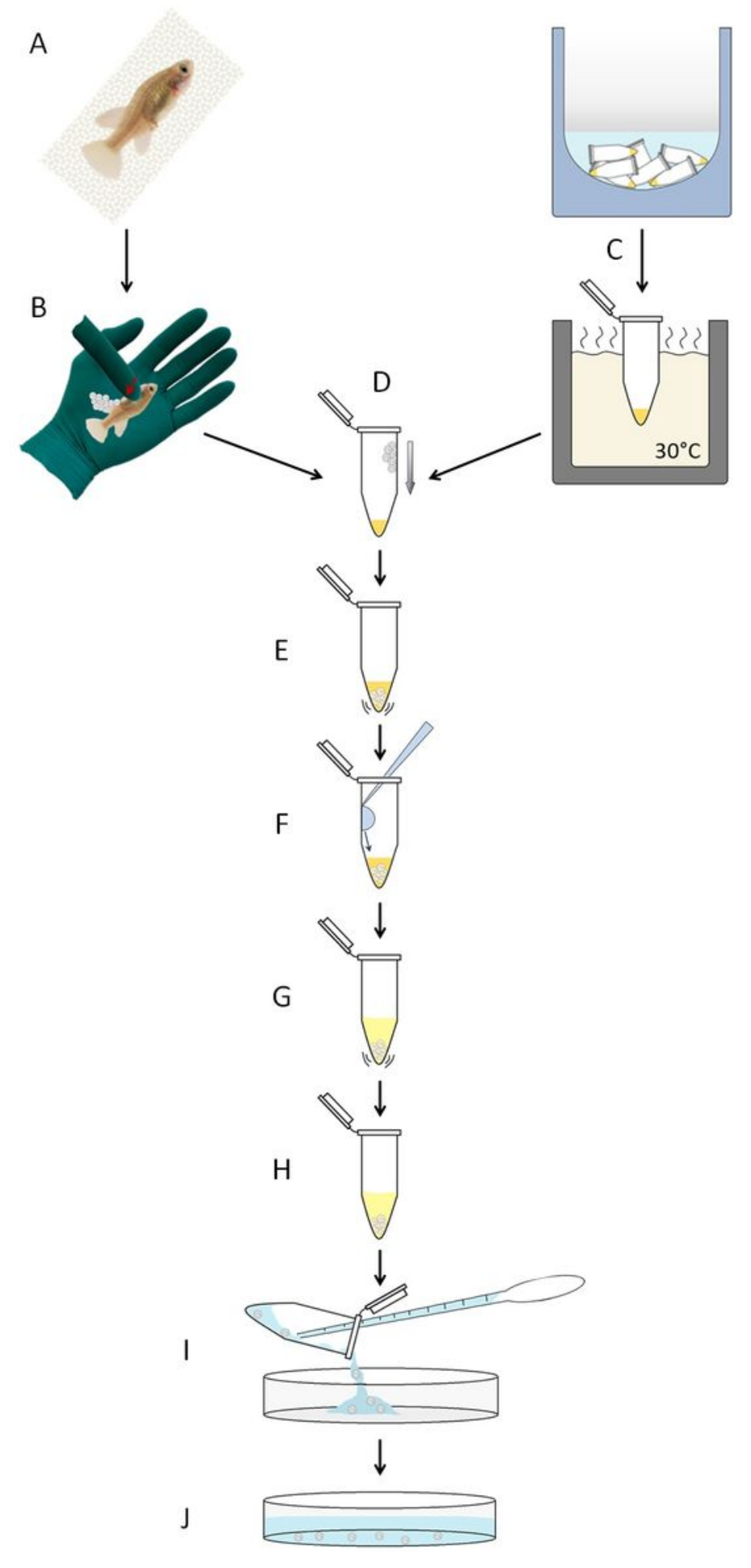

\section{Figure 5}

Schematic of sperm thawing and egg fertilization procedure. (A) Female preparation. (B) Egg collection through gentle abdominal squeezing. (C) Frozen sperm thawing in a water bath. (D-E) Eggs mixed with sperm-extender solution. (F) Activating solution addition and (G) mix. (H) Fertilization. (I) Egg recovery in a petri dish. $(\mathrm{J})$ Fertilized eggs developing in a petri dish. For detailed explanation, refer to the protocol 

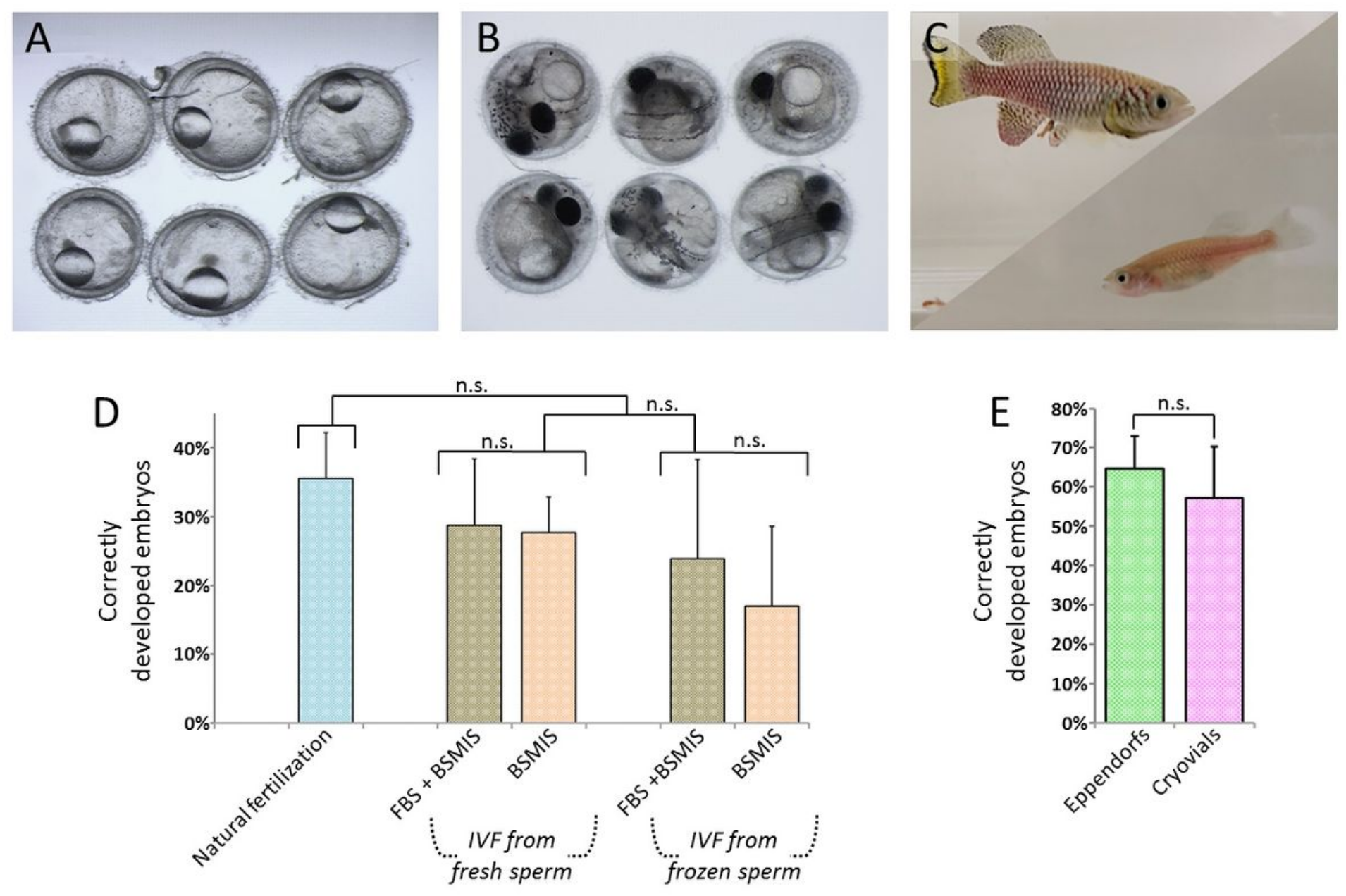

Figure 6

Fertilization rates and embryonic development. (A) Embryos fertilized with IVF using frozen sperm develop through diapause II/mid-somitogenesis stage and later (B), up to advanced developmental stage. (C) Adult fish derived from IVF embryos. (D) Fertilization efficiencies of natural breeding, IVF with fresh extracted sperm and IVF with sperm frozen for 2 weeks. None of the condition tested resulted in fertilization rates significantly different from natural fertilization when tested with one way Anova post hoc Tukey's test. $N=6,8,4,10,3$ independent fertilization trials for each fertilization condition, respectively, in order of appearance in the graph. $\mathrm{N}=1023,440,349,485,201$ total eggs used for fertilization in the trials for each fertilization condition, respectively, in order of appearance in the graph. (E) Fertilization efficiencies of IVF using sperm frozen for 50 days in Cryovials or Eppendorf tubes. The egg pool used for fertilization derived from 10 weeks old females. 3 independent trials were performed using different females and sperm aliquots. $N=45$ total eggs used for cryovials (fertilized with 3 different sperm aliquots) IVF and 52 total eggs for Eppendorfs (fertilized with 3 different sperm aliquots) tests. T-Test between the two groups results was not significant. 

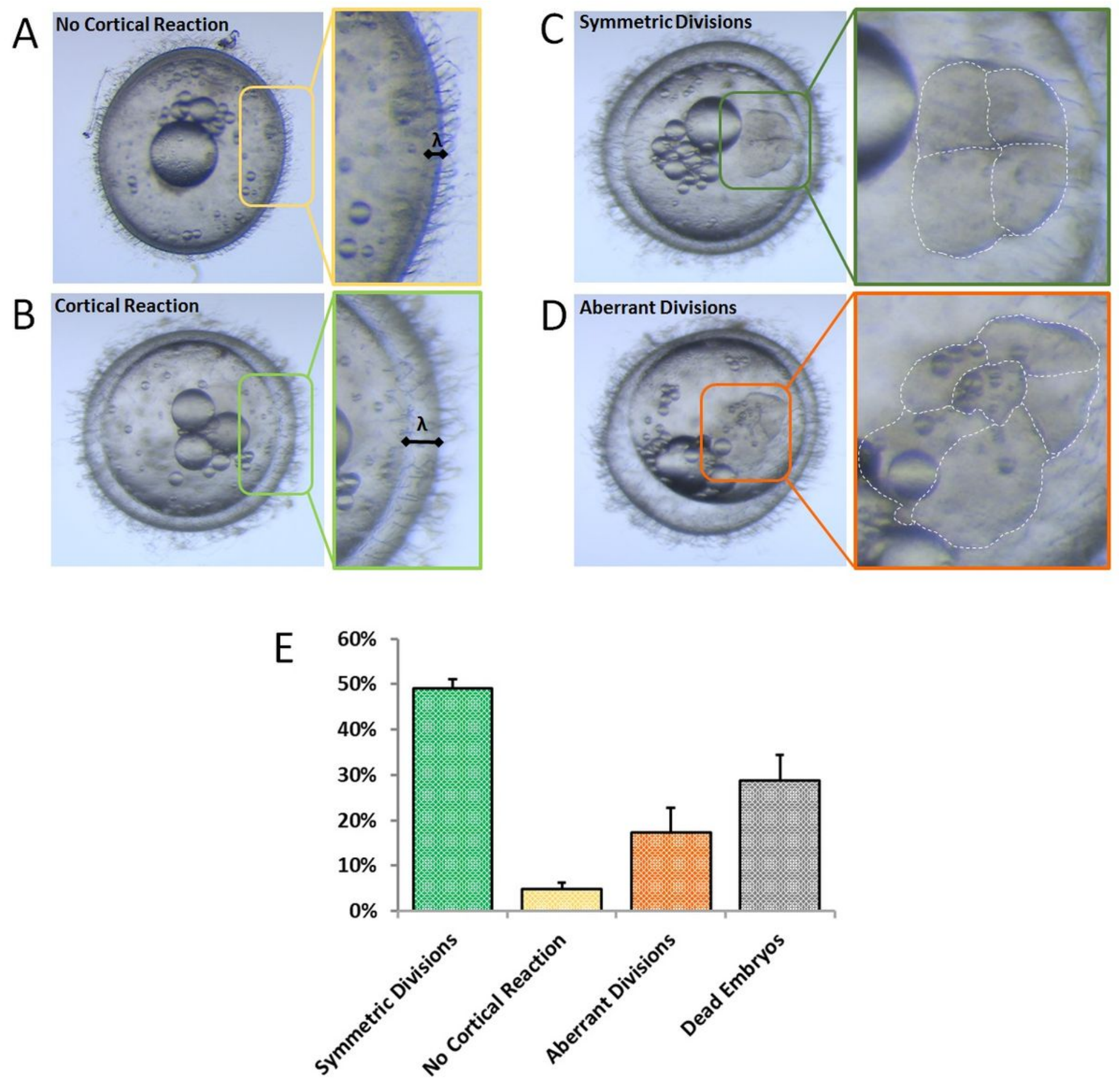

\section{Figure 7}

Cortical reaction and cell division in fertilized and unfertilized eggs. (A) Oocytes that do not undergo the cortical display closely apposed chorion membrane and yolk ( $\lambda$ represent the average distance of $35.0 \pm$ $10.6 \mu \mathrm{m} . \mathrm{N}=8$ ). (B) Embryo in which the cortical reaction has occurred properly where the yolk detaches from the chorion membrane ( $\lambda$ represent the average distance of $87.6 \pm 11.2 \mu \mathrm{m}$. $N=14$ ). (C) Embryo undergoing correct cell division at the 4 cell stage. (D) Pseudoembryo undergoing aberrant divisions, due to missing or incorrect fertilization. Cells are odd in number and of irregular size and shape during the earliest stages. (E) Average embryonic phenotypic distribution. $N=4$ independent natural fertilizations 
with 470, 332, 101, 612 eggs collected, respectively. Embryos were screened after 6 hours from collection and phenotype was assessed by eye. The graph represents the average of the 4 different collections.

\section{Supplementary Files}

This is a list of supplementary files associated with this preprint. Click to download.

- FigureS1.jpg

- S1filelVFprotocol.pdf 